\section{Cellulite durch lokalen Adiponectinmangel?}

Das subkutane Fettgewebe ist kein inerter Energiespeicher, sondern ein endokrin aktives Gewebe. Für das von Adipozyten produzierte Gewebshormon Adiponectin wurden unter anderem antientzündliche und gefäßerweiternde Eigenschaften nachgewiesen. Um einer möglichen Rolle von Adiponectin bei der Entstehung der Cellulite auf die Spur zu kommen, wurden Frauen mit und ohne "Orangenhaut" verglichen.

$\mathrm{D}$ as Autorenteam untersuchte die Expression von Adiponectin im subkutanen Fettgewebe am Gesäß. 15 Probandinnen wiesen eine Cellulite auf, 15 bezüglich Alter, BMI und Rauchstatus vergleichbare Probandinnen zeigten keine Cellulite. In die Studie wurden nur normalgewichtige Frauen eingeschlossen, um eine adipositasbedingte Absenkung des Adiponektinspiegels auszuschließen. Die lokale Genexpression bestimmten die Autoren mit einer Reverse-Transkription-PCR (RTPCR). Mit einem Antikörpertest wurde der Adiponektinspiegel im Plasma bestimmt.

Im subkutanem Fettgewebe aus Cellulitearealen waren signifikant weniger Adiponectin-mRNA nachweisbar. Dagegen bestand im Hinblick auf die Adipo-
nectin-Plasmaspiegel kein Unterschied zwischen Frauen mit und ohne Cellulite.

Die verminderte Adiponectinexpression in Cellulitegewebe wurde von den Studienautoren um Enzo Emanuele, Pavia, so gedeutet: „ Adiponectin könnte als lokaler parakriner Faktor wirken, der das Erscheinungsbild der Haut beeinflusst." Dass die Plasmaspiegel bei Cellulite nicht verändert seien, spreche für eine auf das betroffene subkutane Fettgewebe begrenzte Wirkung, die keine systemische Veränderungen nach sich ziehe.

Auf die Frage, wie die lokal reduzierte Adiponectinsekretion die Entstehung von Cellulite begünstigen könnte, sahen die Studienautoren drei mögliche Mechanismen:
1. Adiponectin ist ein Vasodilatator. Wenn Adiponectin fehlt, kann die Mikrozirkulation in Mitleidenschaft gezogen werden.

2. Adiponectin wirkt antiinflammatorisch. Durch einen Mangel können lokale Entzündungsprozesse entfacht werden.

3. Adiponectin hat antifibrotische Effekte. Vergrößerte Bindegewebssepten sind ein Hauptcharakteristikum der Cellulite.

Fazit: Bei Frauen mit Cellulite wird im subkutanen Fettgewebe des Gesäßes weniger Adiponectin produziert. Die lokal verminderte Adiponektinsekretion könnte bei der Entstehung der Cellulite eine Rolle spielen. Darüber hinaus liefert sie möglicherweise einen Schlüssel zur Behandlung dieser komplexen kosmetischen Störung. Dr. Beate Schumacher

Emanuele E et al. Adiponectin expression in subcutaneous adipose tissue is reduced in women with cellulite. Int J Dermatol 2011; 50: 412-16

Die klinische Untersuchung belegte bei allen vier Patienten eine vollständige Rückbildung der Läsion, die auch in den drei histologischen Präparaten bestätigt werden konnte. Während der durchschnittlichen Beobachtungszeit von 14 Monaten zeigte sich bei keinem Patienten ein Rezidiv.

Fazit: In der Kombinationsbehandlung verbesserte sich die Effektivität der photodynamischen Therapie, die erforderliche Strahlendosis konnte reduziert werden. Allerdings räumen die Autoren ein, dass langfristigere Beobachtungen mit höheren Patientenzahlen erforderlich sind, um den Erfolg dieser Kombinationstherapie sowie mögliche Nebenwirkungen sicher beurteilen zu können.

Dr. Christine Starostzik

den: Mit dem dringen die Strahlen tiefer in die Haut ein. Die Strahlentherapie mit Elektronen lässt sich besser einstellen als die Applikation von Gamma- oder Röntgenstrahlen. Die Gesamtdosis von üblicherweise 10-70 Gy konnte auf 12 Gy reduziert werden.
Nakano A et al. Treatment efficiency of combining photodynamic therapy and ionizing radiation for Bowen's disease. J Eur Acad Dermatol Venereol 2011; 25: 475-8 